\title{
Quantifying the Effect of Atmospherically-Induced Pointing Errors in Optical Geostationary Satellite Feeder Links Using Transmitter Diversity
}

\author{
Ahmad Mustafa \\ Dirk Giggenbach \\ Juraj Poliak \\ Institute of Communications and Navigation (IKN) \\ German Aerospace Center (DLR) \\ 82234 Wessling, Germany \\ ahmad.mustafa@dlr.de
}

\author{
Stephan ten Brink \\ Institute of Telecommunications \\ University of Stuttgart \\ Pfaffenwaldring 47, 70569 Germany
}

\begin{abstract}
Optical links to geostationary (GEO) satellites suffer from atmospherically-induced beam wander which leads to pointing errors at the satellite causing deep fades. In this paper, we show the benefit of transmitter diversity in reducing the fades caused by beam wander. We derive an analytical expression for the reduction of overall scintillation index for a given number of transmitted beams with Gaussian profile in a multiple-input single-output (MISO) system considering solely the effect of beam wander. The transmitted power, beam divergence angle and pointing jitter are kept as free parameters as in the real situation. Moreover, the optimized ratio of transmitted powers between multiple beams is obtained though simulations for a two-fold transmitter diversity to obtain minimum overall power scintillation index (PSI).
\end{abstract}

Keywords-Optical GEO feeder link; Pointing errors, Transmitter diversity; Power scintillation index, Probability density function

\section{INTRODUCTION}

There is an ever-growing demand of data and multimedia services and reaching remote areas on Earth where a terrestrial cable network cannot be established [1], [2]. Communications from geostationary GEO satellites can effectively cover such gaps in the internet connectivity since it is not obstructed by any topological issues on ground. However, ground-to-satellite space links, due to atmospheric index-of-refraction turbulence (IRT), suffer from intensity and phase fluctuations, also called scintillation. Another effect due to atmospheric turbulence is the loss of direct line of sight between the optical ground station (OGS) and the GEO satellite. This is referred to as beam wander which is beam displacement produced by largescale turbulence structures, which appear close to the ground transmitter in a satellite uplink scenario. These structures change the beam path direction, producing a wandering of the beam around the satellite receiver. If the angular beam wander displacement is greater than the beam divergence, then pointing errors lead to deep fades at the satellite, hence loss of signal availability [3].
The OGS establishes bidirectional links with the GEO satellite using the downlink incoming signal to point the uplink beam, the so-called pointing-by-tracking [4]. If both uplink and downlink travel through the same atmosphere, by tracking the downlink signal, the beam wander should be completely precompensated. But in reality, beam wander cannot be perfectly compensated because of the point-ahead angle (PAA). One major issue, in satellite uplinks, is the relationship between isoplanatic angle (IPA) and PAA, when the uplink pointing uses the downlink tracking angle to also compensate the beam wander. The PAA is needed for compensating the movement of the satellite during the time needed by the light to reach it from the ground station. The IPA is defined as the cone in which the atmospheric turbulence can be assumed constant. If uplink and downlink beams travel through the same atmosphere, the angle-of-arrival fluctuations of the downlink beam could be used to pre-correct the uplink beam wander (also assuming same beam size). Due to the PAA, uplink and downlink beams will not cross the same atmosphere. So the residual pointing error is taken into account as the method of "pointing-by-tracking" the transmitted signal cannot ensure a zero steady-state pointing error. Due to these realistic scenarios for an uplink, we have considered an untracked beam for our analysis.

The beam wander can be reduced by increasing the beam divergence at the expense of reduced mean power. The analysis of the variations in optical irradiance due to beam wander in a single-input single-output (SISO) system in [5] highlights the detrimental effects of beam wander for a small beam. A viable solution is to exploit the benefit of transmitter diversity in reducing the fades caused by beam wander. A multiple-input single-output (MISO) system is considered as a fading mitigation scheme which can also effectively reduce the influence of beam wandering in the single photodetector at the satellite receiver. Multiple beams from separate transmitters at the ground station are transmitted through statistically independent fading channels and are combined in a single receiver at the satellite. 
In this work, it is assumed that the optical beam possesses a Gaussian profile. Therefore, for a short-exposure model, one can assume that the Gaussian beam profile does not change in the plane of observation [6].

We derive an analytical expression for the reduction of overall scintillation index for a given number of transmitted beams in a MISO system considering solely the effect of beam wander. The analysis is carried out by means of combining the beta probability density function (PDF) of the multiple optical signals at the receiver in the presence of atmosphericallyinduced beam wander. The PDF for a SISO system is taken from [6], [7], [8], and [9]. In our analysis, the beam divergence, beam wander and transmitted power of each beam are set as free parameters. The analytical results are simulated using realistic parameters for the GEO uplink. This analysis is helpful in choosing the values of these free parameters to obtain the desired gain and overall power scintillation index (PSI).

The remainder of this paper is organized as follows: The theoretical derivation of PSI, of $n$-fold transmitter diversity considering only pointing errors, is presented in Section II. In Section III, the simulations of the performance parameters for evaluating transmitter diversity is given. Section IV includes the results and discussion of the performance parameters. Finally, Section V concludes the paper.

\section{DeRiVATION OF POWER SCINTILLATION INDEX DUE TO POINTING ERRORS IN MULTIPLE BEAMS}

In the absence of jitter and assuming a Gaussian beam, the irradiance $I_{\mathrm{r}}$ seen by the satellite receiver, after the optical wave propagates through the turbulent atmosphere in a FSO link at a propagation distance $Z$ in the far field and in the direction $\alpha$ from the optical axis (OA) can be expressed as [11]

$$
I_{\mathrm{r}}(\alpha, Z)=\frac{2 P_{\mathrm{t}}}{\pi\left(\omega_{0} Z\right)^{2}} \exp \left(-2 \frac{\alpha^{2}}{\omega_{0}^{2}}\right)
$$

where $\omega_{0}$ is the $1 / e^{2}$ beam intensity divergence half-angle and $P_{\mathrm{t}}$ is the transmitted power. The first term in (1) is the axial intensity and the exponential term is the Gaussian function with its peak at $\alpha=0$ (on axis) and drops monotonically with increasing $\alpha$.

In the presence of pointing errors, the pointing PDF with angular random jitter of $\sigma_{j}$ due to turbulence is the NakagamiRice distribution and is given by [6], [7], and [10]

$$
p_{j}(\alpha, \varphi)=\frac{\alpha}{{\sigma_{j}}^{2}} \exp \left(-\frac{\alpha^{2}+\varphi^{2}}{2{\sigma_{j}}^{2}}\right) I_{0}\left(\frac{\alpha \varphi}{{\sigma_{j}}^{2}}\right)
$$

where $\varphi$ is the bias beam-pointing error angle from the OA and $I_{0}(\cdot)$ is a modified Bessel function of the first kind and order zero. We assume that the bias error angle can be regarded as zero in this paper and now the pointing PDF reduces to the Rayleigh distribution

$$
p_{j}(\alpha, 0)=\frac{\alpha}{{\sigma_{j}}^{2}} \exp \left(-\frac{\alpha^{2}}{2{\sigma_{j}}^{2}}\right)
$$

We consider the intensity $I$ which is normalized to the axial intensity. This $I$ is given by the exponential term in (1). Therefore, the PDF of $I$ becomes the beta distribution and is given according to $[6,8]$

$$
\begin{gathered}
p(I)=\beta I^{(\beta-1)} \quad \text { for } 0 \leq I \leq 1 \\
\bar{I}=\frac{\beta}{\beta+1} \\
\operatorname{var}(I)=\frac{\beta}{(\beta+2)(\beta+1)^{2}}
\end{gathered}
$$

where $\bar{I}$ is the average value, $\operatorname{var}(I)$ is the variance, and

$$
\beta=\omega_{0}^{2} / 4 \sigma_{j}^{2}
$$

The scintillation index analysis presented in this paper takes the beta PDF in (4) into account to come up with an overall scintillation index for the combination of $N$ transmitted beams in a MISO system considering pointing errors only. We define PSI as power variance normalized by the square of the mean value. We include a power scaling factor $p$ for the transmitted power of each beam and now we can consider $I_{m}=p_{m} I$ where $p_{m}$ is the power scale factor of the $m$ th beam. PSI for a SISO system with pointing errors is:

$$
\begin{aligned}
& P S I_{\text {SISO }}=\frac{\operatorname{var}\left(I_{m}\right)}{\operatorname{mean}\left(I_{m}\right)^{2}} \\
& =\frac{E\left\{I_{m}{ }^{2}\right\}-\left(E\left\{I_{m}\right\}\right)^{2}}{\left(E\left\{I_{m}\right\}\right)^{2}}
\end{aligned}
$$

$E\{\cdot\}$ is the expected value of the random intensity $I$ when it follows beta distribution. Using definition of $E\{\cdot\}$, we can write (6) as

$$
P S I_{\mathrm{SISO}}=\frac{\int_{0}^{1} I_{m}{ }^{2} p(I) d I-\left(\int_{0}^{1} I_{m} p(I) d I\right)^{2}}{\left(\int_{0}^{1} I_{m} p(I) d I\right)^{2}}
$$

The integration limits vary from 0 to 1 because of normalization of received beam irradiance. The mean and variance of the beam is now given as $\overline{I_{m}}=p_{m} \bar{I}$ and $\operatorname{var}\left(I_{m}\right)=p_{m}{ }^{2} \operatorname{var}(I)$ respectively. After solving (6), the final PSI of a SISO system with pointing errors comes out to be

$$
P S I_{\text {SISO }}=\frac{1}{\beta(\beta+2)}
$$


Equation (7) can be extended to include the effect of multiple incoherent beams with pointing errors. It can be written as

$$
P S I_{\mathrm{MISO}}=\frac{\sum_{m=1}^{N} \operatorname{var}\left(I_{m}\right)}{\left(\sum_{m=1}^{N} \overline{I_{m}}\right)^{2}}
$$

which yields

$$
P S I_{\text {MISO }}=\frac{\sum_{m=1}^{N} \frac{p_{m}{ }^{2} \beta_{m}}{\left(\beta_{m}+1\right)^{2}\left(\beta_{m}+2\right)}}{\left(\sum_{m=1}^{N} \frac{p_{m} \beta_{m}}{\beta_{m}+1}\right)^{2}}
$$

$p_{m}$ and $\beta_{m}$ are the power scaling factor and beta of the $m$ th beam.

\section{SIMULATION PROCEDURE FOR EVALUATING TRANSMITTER DIVERSITY}

To verify the scintillation index of the SISO and MISO system as given by the expressions in (7) and (8) respectively, we perform numerical simulations for an optical signal with pointing errors in the uplink of a ground-GEO satellite channel. We assume symmetric Gaussian functions along $x$ and $y$ direction from OA. The radial angle $\alpha$ in (1) becomes a random variable in the presence of pointing errors with angular jitter $\sigma_{j, x}$ and $\sigma_{j, y}$ in $x$ and $y$ direction, respectively. The $\sigma_{j, x}$ and $\sigma_{j, y}$ are used in (1) to generate the irradiance seen at the satellite. The $\omega_{0}$ and $\sigma_{j}$ define the $\beta$ value of the beam with received irradiance $I_{m}=p_{m} I$ as described in Section II. The $\omega_{0}$ for all beams is kept the same assuming the collimator and beam size is fixed at the ground station. The performance parameters to evaluate the benefit of transmitter diversity in mitigating fades due to the pointing errors are taken as PSI and PDF. The results are presented and discussed in Section IV.

\section{A. PSI}

PSI for the beta distributed intensity vector is found by numerically calculating the variance and mean and using the definition of PSI in (6). PSI for this beam is also calculated using (7) which depends on the value of $\beta$ only. To observe the benefit of transmitter diversity and to verify (8), we simulate 4 uplink beams as in the SILEX setup at the OGS in Tenerife [12]. The beams are simulated with both equal and unequal values of $\beta$ as in the real case. Also, the simulations are done taking unequal transmitted powers and when the powers are reduced equally by the factor $N$. In the latter case, the overall scintillation index is expected to reduce by $N$ when all beams have same values of $\beta$ [13].

\section{B. $P D F$}

Besides that PDF of a 4x1 MISO system is compared with that of a SISO system. The PDFs are obtained from the histogram of the received intensities.

\section{Influence of Unequal Transmitted Powers}

Simulations are done to find the optimum transmitted power for each beam to get minimum PSI using (8) for given values of $\beta$. Currently, this optimization is done for 2-Tx diversity as an example. Two cases are simulated and in each case a unique $\beta$ value of one of the beams $\beta_{1}$ is fixed and the $\beta$ value of the second beam $\beta_{2}$ is varied along with the transmit powers to find the combined PSI values. In the simulations the total transmitted power is normalized to one.

\section{RESULTS AND DISCUSSION}

We present and discuss the results in this section to show the benefits of using multiple beams in reducing the fades caused by pointing errors only. For this purpose, four cases are presented in Table I.

In case I, the transmitted power of each beam is equally reduced by $N$ and equal pointing jitter is assumed. This case is taken as a benchmark. The sum of power of transmitted beams in all cases is the same as the individual beam without transmitter diversity for a fair comparison. The value of $\beta$ from (6) is 2.93 as an example which is taken using $\omega_{0}$ and $\sigma_{j}$ values from [14].

In case II, the transmitted power of each beam is left unchanged while $\sigma_{j}$ is varied to see the effect of jitter on individual and combination of beams.

In case III, jitter for individual beams is kept the same and the transmitted powers are changed.

Finally, in case IV, both transmitted powers and jitters are varied to find the combined effect on the overall PSI.

The effect of the power and jitter values on the selected performance parameters is presented next:

\section{A. PSI}

In the first case, the PSI of a MISO system reduces by a factor equals to the number of transmitters because of combination of equal irradiance from all beams at the receiver. This is in accordance with the known theory [13]. The PSI of a SISO and MISO system varies according to (7) and (8). It is seen, that PSI for the SISO system is independent of beam intensity. The PSI for a MISO system changes due to change in irradiance statistics when multiple incoherent beams are combined. As shown later, the overall scintillation index can be controlled by selecting appropriate transmitted power according to the pointing jitter associated with each beam.

\section{B. $P D F$}

The PDFs for the SISO and MISO system with the four cases in Table 1 are presented in Fig. 1-4. The received intensity for the SISO system from (1) is normalized to the maximum transmitted power of one transmitter from case I while the received intensity for MISO system is normalized to the total transmitted power of all four transmitters. The improvement in the PDF of a MISO system depends on the 
pointing jitter values and transmitted power associated with each beam.

TABLE I. COMPARISON BETWEEN THEORETICAL AND SIMULATED PSI FOR SISO AND MISO SYSTEM

\begin{tabular}{|c|c|c|c|c|c|c|}
\hline \multirow{2}{*}{$\begin{array}{c}\text { Beam } \\
\#\end{array}$} & $\begin{array}{c}p_{m} \\
(\%)\end{array}$ & $\begin{array}{c}\sigma_{j} \\
(\mu \mathrm{rad})\end{array}$ & $\beta$ & PSI $_{\text {IISo }}$ & $\begin{array}{c}\text { From } \\
\text { Simulations }\end{array}$ & $\begin{array}{c}\text { From } \\
(8)\end{array}$ \\
\hline
\end{tabular}

\begin{tabular}{|c|c|c|c|c|c|}
\hline \multicolumn{6}{|c|}{ Case I } \\
\hline 1 & 25 & 1.87 & 2.93 & 0.069 & \multirow{4}{*}{0.017} \\
\hline 2 & 25 & 1.87 & 2.93 & 0.069 & \\
\hline 3 & 25 & 1.87 & 2.93 & 0.069 & \\
\hline 4 & 25 & 1.87 & 2.93 & 0.070 & \\
\hline
\end{tabular}

\begin{tabular}{|c|c|c|c|c|c|}
\hline \multicolumn{6}{|c|}{ Case II } \\
\hline 1 & 25 & 1.87 & 2.93 & 0.070 & \multirow{4}{*}{0.018} \\
\hline 2 & 25 & 2.01 & 2.53 & 0.087 & \\
\hline 3 & 25 & 2.37 & 1.82 & 0.144 & \\
\hline 4 & 25 & 1.27 & 6.35 & 0.019 & \\
\hline
\end{tabular}

Case III

\begin{tabular}{|l|l|l|l|l|l|l|}
\hline $\mathbf{1}$ & 25 & 1.87 & 2.93 & 0.069 & & \\
\hline $\mathbf{2}$ & 30 & 1.87 & 2.93 & 0.069 & \multirow{2}{*}{0.020} & \multirow{2}{*}{0.012} \\
\hline $\mathbf{3}$ & 35 & 1.87 & 2.93 & 0.070 & & \\
\hline $\mathbf{4}$ & 10 & 1.87 & 2.93 & 0.070 & & \\
\hline
\end{tabular}

\begin{tabular}{|l|l|l|l|l|l|l|}
\hline \multicolumn{7}{|c|}{ Case IV } \\
\cline { 1 - 4 } $\mathbf{1}$ & 25 & 1.87 & 2.93 & 0.069 & & \\
\cline { 1 - 5 } $\mathbf{2}$ & 30 & 2.01 & 2.53 & 0.087 & \multirow{2}{*}{0.027} & 0.027 \\
\hline $\mathbf{3}$ & 35 & 2.37 & 1.82 & 0.142 & & \\
\cline { 1 - 4 } $\mathbf{4}$ & 10 & 1.27 & 6.35 & 0.019 & & \\
\hline
\end{tabular}

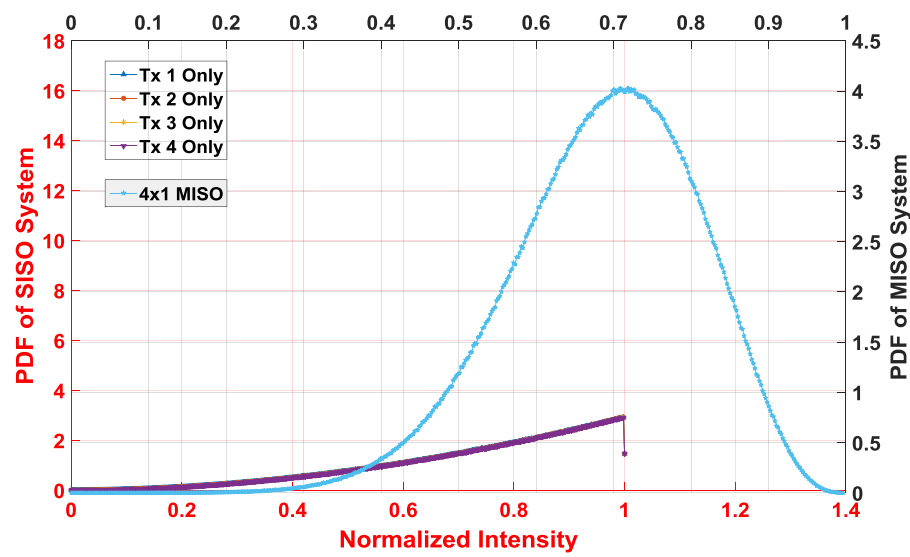

Fig. 1: Case-I -- Equal transmit powers and equal $\beta$ values

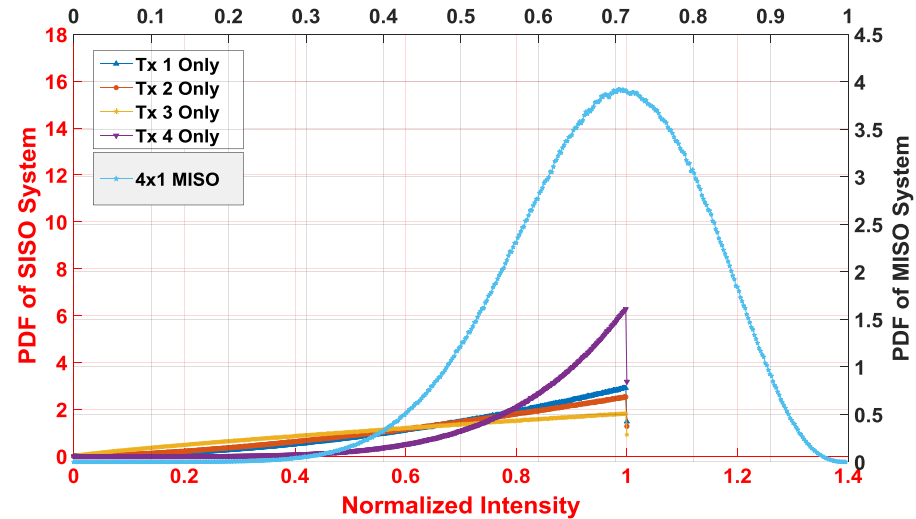

Fig. 2: Case-II -- Equal transmit powers and unequal $\beta$ values

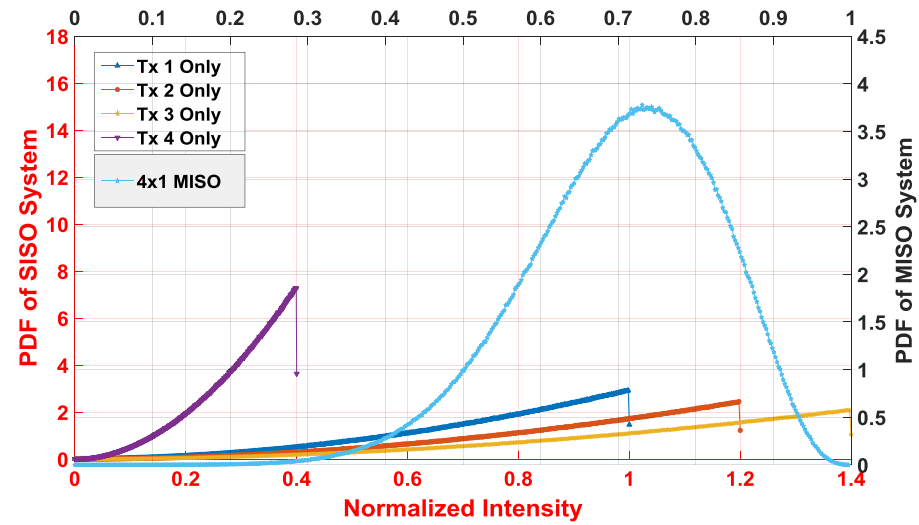

Fig. 3: Case-III -- Unequal transmit powers and equal $\beta$ values

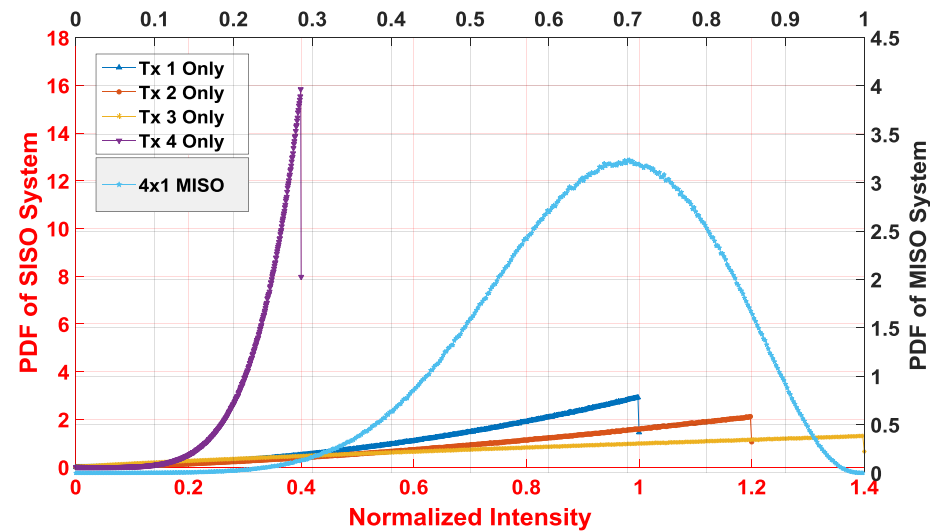

Fig. 4: Case-IV -- Unequal transmit powers and unequal $\beta$ values

\section{Influence of Unequal Transmitted Powers}

The influence of transmitted powers on the combined PSI can be seen in the contour plots in Fig. 5 and Fig. 6 where values of $\beta_{1}$ are fixed to 2.93 and 5 , respectively. For each case and a $\beta_{2}$ value, there are optimum transmit powers which yield in minimum PSI as shown by the red curve in the contour plots. From the plots we deduce that the beam with higher $\beta$ value should have more transmit power to get the minimum combined PSI. The color bar represents PSI values in logarithmic scale. 


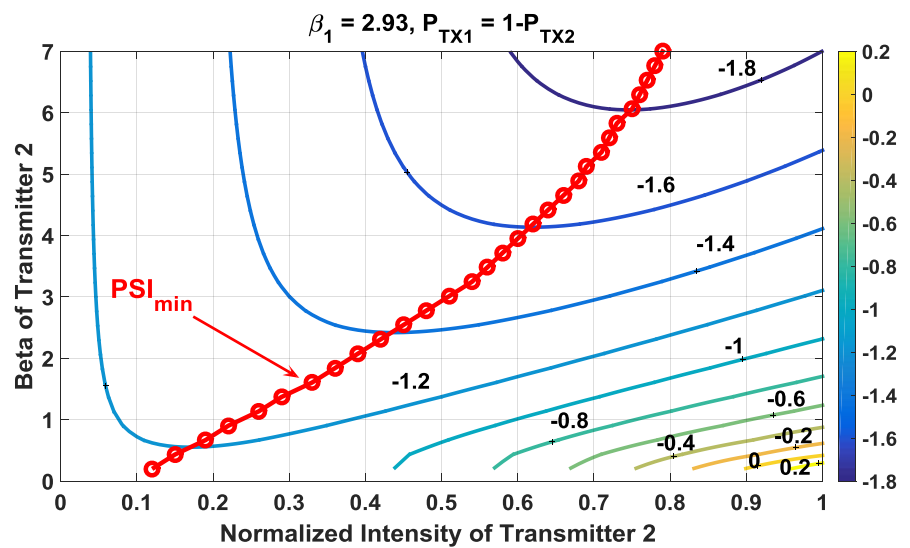

Fig. 5: Contour plot displaying the isolines of PSI variations in logarithmic scale

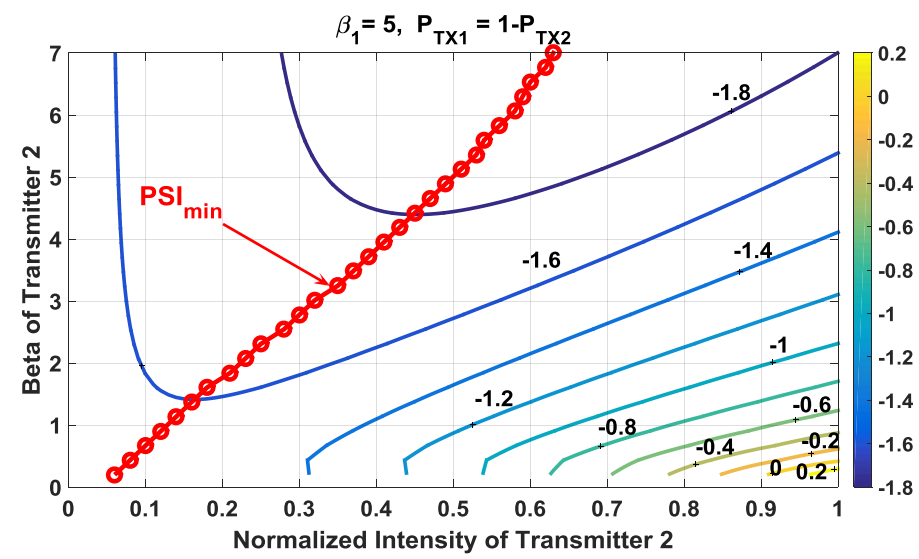

Fig. 6: Contour plot displaying the isolines of PSI variations in logarithmic scale

Table II. REQUIRED TRANSMIT POWERS FOR MINIMUM PSI IN A 2x1 MISO SYSTEM

\begin{tabular}{|c|c|c|c|c|c|}
\hline Nr. & $\boldsymbol{\beta}_{\mathbf{1}}$ & $\boldsymbol{\beta}_{\mathbf{2}}$ & $\boldsymbol{P}_{\mathbf{T} \mathbf{1}}$ & $\boldsymbol{P}_{\text {Tx2 }}$ & $\boldsymbol{P S}_{\text {min }}$ \\
\hline \multicolumn{6}{|c|}{ Case I } \\
\hline $\mathbf{1}$ & 2.93 & 0.20 & 88 & 12 & 0.067 \\
\hline $\mathbf{2}$ & 2.93 & 2.93 & 50 & 50 & 0.035 \\
\hline $\mathbf{3}$ & 2.93 & 7 & 21 & 79 & 0.013 \\
\hline \multicolumn{7}{|c|}{ Case II } & & \\
\hline $\mathbf{1}$ & 5 & 0.20 & 94 & 6 & 0.028 \\
\hline $\mathbf{2}$ & 5 & 5 & 50 & 50 & 0.014 \\
\hline $\mathbf{3}$ & 5 & 7 & 37 & 63 & 0.010 \\
\hline
\end{tabular}

Also, we see the effect of increasing the $\beta_{1}$ on combined PSI in Fig. 6. When comparing it with case I in Fig. 5, it is seen the PSI values can be further reduced for the same $\beta_{2}$ if $\beta_{1}$ is increased. However, in this case, the transmit powers for each of the two beams are changed. As an example, for each case of $\beta_{1}$, three different values of $\beta_{2}$ along with the associated transmit powers to obtain minimum PSI are given in Table II.

\section{CONCLUSION}

In this paper, we derive the analytical expression for the scintillation index considering the combined effect of multiple beams with pointing errors only. This expression includes the transmitted power, beam divergence and jitter as free parameters as it is in the case of real uplink. We then verify the theoretical expression with simulations and find the results supporting each other. The performance of transmitter diversity is evaluated in terms of PSI and PDF. Also, it is shown for a 2Tx diversity that the transmitted powers of each beam can be optimized to achieve minimum PSI for given $\beta$ values associated with each beam. Transmitter diversity is shown to be an attractive scheme to mitigate the effect of atmospherically-induced pointing errors. Using the analysis presented in this paper, we can say that the desired performance in the GEO uplink can be achieved by proper selection of number of transmitters, beam divergence, and transmitted power of each beam to reduce the effect of beam wander and to achieve the desired signal quality at the satellite receiver.

\section{REFERENCES}

[1] M. A. Khalighi, M. Uysal, "Survey on free space optical communication: A Communication theory perspective," IEEE Comm. Surve. \& Tut., vol. 16, no. 4, pp. 2231-2258, 2014.

[2] L.C. Andrews, R.L. Phillips, "Laser beam propagation through random media", SPIE-Press 2005.

[3] A. Rodriguez -Gomez, F. Dios, J.A. Rubio, A. Comeron, "Temporal statistics of the beam-wander contribution to scintillation in ground-tosatellite optical links: an analytical approach", Applied Optics, Vol. 44, No. 21, July 2005.

[4] R. Mata-Calvo, P. Becker, D. Giggenbach, F. Moll, M. Schwarzer, M Hinz, Z. Sodnik, "Transmitter diversity verification on ARTEMIS geostationary satellite“, SPIE Photonics West, Feb. 2014.

[5] F. Dios, J. Antonio Rubio, A. Rodri'guez, and A. Comero'n, "Scintillation and beam-wander analysis in an optical ground stationsatellite uplink", Applied Optics, Vol. 43, No. 19/ July 2004.

[6] K. Kiasaleh and T.-Y. Yan, "A statistical model for evaluating GOPEX uplink performance," TDA Progress Report 42-11 1, Jet Propulsion Laboratory (1992).

[7] M. Toyoshima and K. Araki, "Effects of time-averaging on optical scintillation in a ground-to-satellite atmospheric propagation," Applied Optics, Vol. 39, No. 12, pp. 1911-1919 (2000).

[8] D. L. Fried, "Statistics of laser beam fade induced by pointing jitter," Applied Optics, Vol. 12, No. 2, pp. 422-423 (1973).

[9] P. J. Titterton, "Power Reduction and Fluctuations Caused by Narrow Laser Beam Motion in the Far Field," Applied Optics, Vol. 12, No. 2, pp. 423-425 (1973).

[10] M. Toyshima, T. Jono, K. Nakagawa, and A. Yamamoto, "Optimum intersatellite link design in the presence of random pointing jitter for free-space laser communication systems", Proceedings of SPIE Vol. 4635 (2002).

[11] B.E.A. Saleh, M.C. Teich "Fundamental of Photonics", New York: Wiley, 1991 
[12] T. T. Nielsen, G. Oppenhaeuser, B. Laurent, and G. Planche, "In-orbit test results of the optical intersatellite link, SILEX. A milestone in satellite communication," in Proc. 53rd Int. Astronautical Congr. Int. Astronautical Fed., IAC-02-M.2.01, Oct. 10-19, 2002.

[13] W-ming Wu, Y. Ning, P-fei Zhang, X-xing Feng, C-hong Qiao, "Scintillation analysis for multiple uplink Gaussian beams in the presence of beam wander", Proc. of SPIE Vol. 9142, 914223, 2014
[14] M. Toyoshima, S. Yamakawa, T. Yamawaki, K. Arai, M.R GarciaTalavera, A. Alonso, Z. Sodnik, and B. Demelenne, "Long-Term Statistics of Laser Beam Propagation in an Optical Ground-toGeostationary Satellite Communications Link", IEEE transactions on antennas and propagation, Vol. 53, No. 2, February 2005 